\title{
Accuracy of Elevation Reduction Factor
}

\author{
Earl F. Burkholder ${ }^{1}$ Member, ASCE
}

\begin{abstract}
When using state plane coordinates or performing other geodetic computations, the ratio $r /(r+h)$ is the elevation factor commonly used to reduce a horizontal distance to its sea level or ellipsoidal equivalent. In most cases, an approximation is used for the earth's radius while the value of elevation is usually much better known. That is as it should be. But the question examined in this paper is, "What is an acceptable approximation for the earth's radius and how accurately must the elevation be known to assure sufficient precision in the answer?" An equation that can be used to answer those questions is derived using concepts of error propagation.
\end{abstract}

\section{Introduction:}

A measured slope distance is typically reduced to horizontal using the slope distance and either: 1) the difference in elevation of the end points; or 2) the vertical angle (zenith direction) of the slope distance as measured from either or both ends (Burkholder 1991). Regardless of how the horizontal distance was obtained, the next step is to reduce the horizontal distance either to sea level or, being more specific, to the ellipsoid using the ratio:

$$
\begin{aligned}
& E F=\frac{r}{r+h} \text { where. } \ldots \\
& \text { EF = elevation factor, } \\
& r=\text { approximate radius of the earth and } \\
& h=\text { elevation of horizontal distance being reduced. }
\end{aligned}
$$

\footnotetext{
${ }^{1}$ Associate Professor, Surveying Engineering Department - 3SUR, New Mexico State University, Las Cruces, New Mexico 88003
} 
The question to be addressed is, "What is an acceptable approximation for the radius of the earth and how accurately must the elevation be known to assure adequate precision in the computed sea level or ellipsoidal distance?" Tools to answers those questions are developed from the following error propagation equation:

$$
{\sigma_{U}}^{2}=\left(\frac{\partial U}{\partial X}\right)^{2} \sigma_{X}^{2}+\left(\frac{\partial U}{\partial Y}\right)^{2} \sigma_{Y}^{2}+\left(\frac{\partial U}{\partial Z}\right)^{2} \sigma_{Z}^{2}+\ldots
$$

where $U=f(X, Y, Z \ldots) ; X, Y$, and $Z$ are independent variables, and $\sigma_{X}, \sigma_{Y}$ and $\sigma_{z}$ are the standard deviations of the variables.

The intent of this article is not to stipulate using one value in preference to another, but to identify a reliable tool that can be used to evaluate the impact of approximations made by the user.

\section{Elements:}

The radius of the earth and the elevation of the horizontal distance are the two elements used to reduce a horizontal distance to sea level. It is left for the reader to decide which definition of horizontal distance will be used in the reduction. Of the various definitions of horizontal distance given in Burkholder (1991):

- $\mathrm{HD}(1)$, Figure $1 \mathrm{a}$, is the simple right triangle component of slope distance. It is used extensively in practice and is sufficiently precise for many applications.

- $\mathrm{HD}(2)$, Figure $1 \mathrm{~b}$, is the tangent plane distance between plumb lines.

- $\mathrm{HD}(3)$, Figure $1 \mathrm{c}$, is the chord distance between plumb lines. It's endpoints are at the same elevation and it is perpendicular to the plumb line only at the midpoint. - $\mathrm{HD}(4)$, Figure 1c, is the arc distance between plumb lines at the same elevation as the endpoints of $\mathrm{HD}(3)$. 
As shown in Burkholder (1991), the differences between $\mathrm{HD}(2), \mathrm{HD}(3)$, and $\mathrm{HD}(4)$ are miniscule. If $\mathrm{HD}(1)$ is not precise enough, either $\mathrm{HD}(2), \mathrm{HD}(3)$, or $\mathrm{HD}(4)$ can generally be used instead.

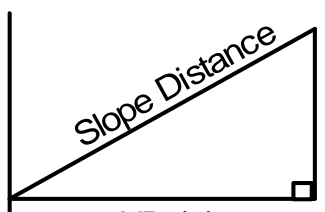

$\mathrm{HD}(1)$

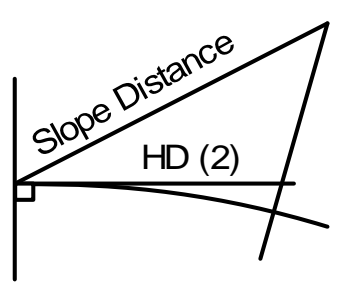

(b)

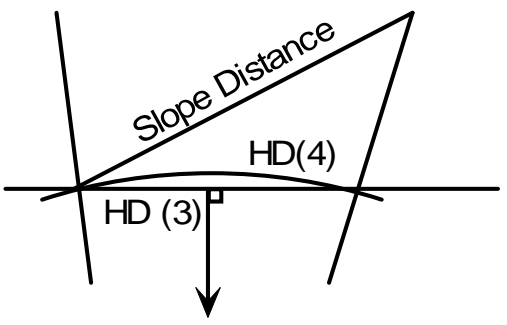

(c)

Figure 1, Slope Distance and Various Definitions of Horizontal Distance

Notes with regard to the elevation factor elements are:

1. When computing the elevation factor, earth's radius is often taken to be $6,372,000$ meters or $20,906,000$ feet. Either value is acceptable and, as implied by rules of significant digits, is accurate to the nearest 1000 feet or 1000 meters.

2. The earth is not quite spherical, but flattened at the poles. Students of geodesy soon learn that the earth's radius in the north-south direction changes from a smaller value at the equator to a larger value at the poles. The geometrical mean radius of the earth at a given latitude is computed as:

$$
\begin{aligned}
& r=\frac{a \sqrt{1-e^{2}}}{1-e^{2} \sin ^{2} \phi} \quad \text { where, for GRS } 1980 \text { (and NAD83); } \\
& \mathrm{a}=\text { semi-major axis (radius of equator) }=6,378,137.000 \mathrm{~m} \\
& \mathrm{e}^{2}=\text { eccentricity squared }=0.00669438002290 . \\
& \phi=\text { geodetic latitude. }
\end{aligned}
$$


Table1 lists values of geometrical mean radius for various values of latitude. Increments of $5^{\circ}$ are used except in those cases needed to match the approximate values.

\section{Table 1, Geometrical Mean Radius of Curvature at Various Latitudes}

\begin{tabular}{ccc}
$\begin{array}{c}\text { Latitude } \\
\text { (degrees) }\end{array}$ & $\begin{array}{c}\text { Mean Radius } \\
\text { (meters) }\end{array}$ & $\begin{array}{c}\text { Mean Radius } \\
\text { (U.S. Survey Feet) }\end{array}$ \\
\hline 0 & $6,356,752.314$ & $20,855,444.88$ \\
5 & $6,357,075.580$ & $20,856,505.47$ \\
10 & $6,358,035.749$ & $20,859,655.62$ \\
15 & $6,359,604.205$ & $20,864,801.46$ \\
20 & $6,361,734.148$ & $20,871,789.45$ \\
25 & $6,364,361.913$ & $20,880,410.71$ \\
30 & $6,367,408.778$ & $20,890,406.96$ \\
35 & $6,370,783.223$ & $20,901,477.96$ \\
$36-43-04$ & $6,372,000.000$ & $20,905,470.00$ \\
$36-56-36$ & $6,372,161.544$ & $20,906,000.00$ \\
40 & $6,374,383.582$ & $20,913,290.14$ \\
45 & $6,378,101.030$ & $20,925,486.46$ \\
50 & $6,381,822.817$ & $20,937,697.02$ \\
55 & $6,385,435.668$ & $20,949,550.19$ \\
60 & $6,388,829.252$ & $20,960,683.97$ \\
65 & $6,391,899.595$ & $20,970,757.25$ \\
70 & $6,394,552.344$ & $20,979,460.48$ \\
75 & $6,396,705.765$ & $20,986,525.50$ \\
80 & $6,398,293.360$ & $20,991,734.13$ \\
85 & $6,399,266.022$ & $20,994,925.27$ \\
90 & $6,399,593.626$ & $20,996,000.09$ \\
& & \\
& &
\end{tabular}

3. Figure 2 illustrates the relationship of horizontal distance, sea level distance and ellipsoid distance. It also shows that geoid height is the difference between the ellipsoid and sea level. Historical practice has included reducing horizontal distances to sea level, but when working with state plane coordinates, or other geodetic computations, it is more appropriate to reduce horizontal distance to the ellipsoid. Equation (6), derived in the next section, can be used to assist in deciding 
whether it matters if one uses ellipsoid height or orthometric height in computing the elevation factor. Additional material on elevations is found in Burkholder (2002).

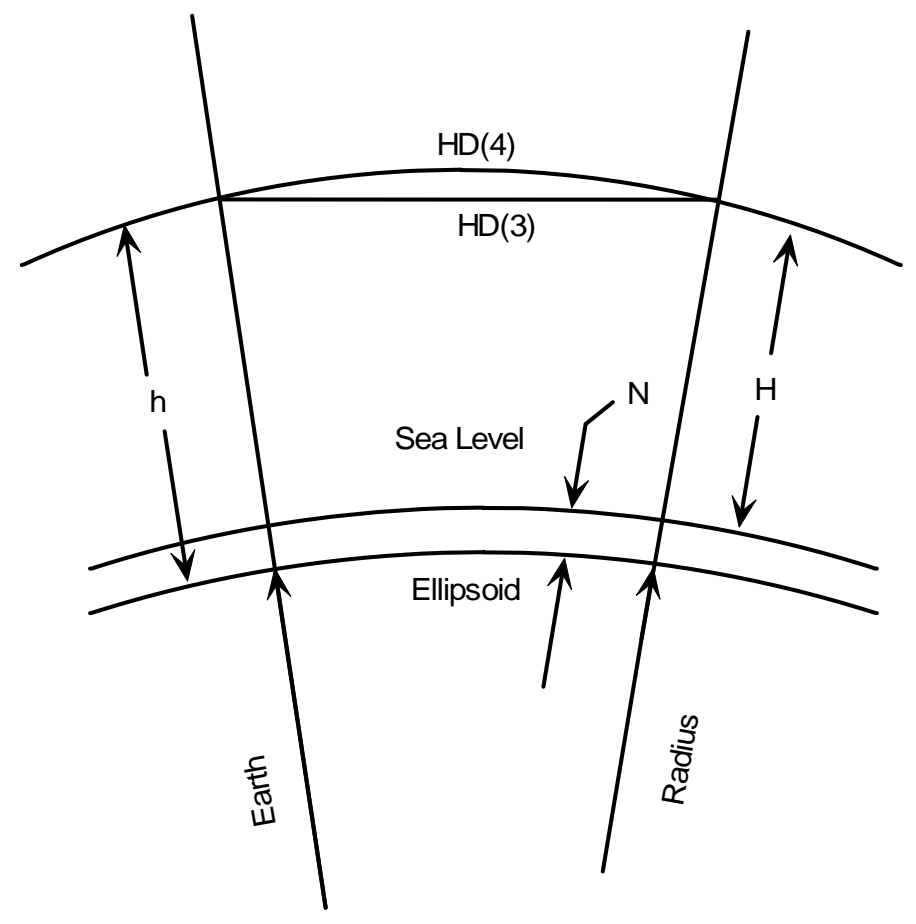

$\mathrm{H}=$ Orthometric height (Nominally elevation)

$\mathrm{h}=$ Ellipsoid height (From GPS data)

$\mathrm{N}=$ Geoid height (From geoid modeling or from $\mathrm{h}-\mathrm{H}$ )

$\mathrm{h}=\mathrm{H}+\mathrm{N}$

Figure 2 Diagram of Elevation Reduction Factor

\section{Derivation:}

The derivation involves taking the partial derivatives of the elevation factor (EF) with respect to each of the two variables, earth radius, $r$, and elevation, $\mathrm{h}$.

$$
\begin{aligned}
E F & =\frac{r}{r+h}=r(r+h)^{-1} \\
\frac{\partial E F}{\partial r} & =(r)(-1)(r+h)^{-2}(1)+\left(\frac{1}{r+h}\right)(1)=\frac{h}{(r+h)^{2}} \\
\frac{\partial E F}{\partial h} & =r(-1)(r+h)^{-2}=\frac{-r}{(r+h)^{2}}
\end{aligned}
$$


Now, substituting equations (4) and (5) into equation (2) along with appropriate standard deviations, the equation for the standard deviation of EF becomes:

$$
\begin{aligned}
\sigma_{E F}^{2} & =\left(\frac{\partial E F}{\partial r}\right)^{2} \sigma_{r}^{2}+\left(\frac{\partial E F}{\partial h}\right)^{2} \sigma_{h}^{2} \\
\sigma_{E F} & =\sqrt{\left[\frac{h}{(r+h)^{2}}\right]^{2} \sigma_{r}^{2}+\left[\frac{-r}{(r+h)^{2}}\right]^{2} \sigma_{h}^{2}}
\end{aligned}
$$

Equation (6) is very powerful in that it can be used to compute the uncertainty (standard deviation) of the elevation factor for any combination of radius and elevation uncertainties selected by the user. Two examples, one in English units and one in metric units, are given next.

\section{English units example:}

$$
\begin{aligned}
& \text { Assume: } \quad \mathrm{r}=20,906,000 \text { feet }+/-1,000 \text { feet } \\
& \qquad \mathrm{h}=3,280 \text { feet }+/-10 \text { feet } \\
& \text { Compute: } \quad E F=\frac{r}{r+h}=\frac{20,906,000}{20,909,280}=0.9998431319 \\
& \sigma_{E F}^{2}=\left[\frac{3,280}{(20,909,280)^{2}}\right]^{2}(1000)^{2}+\left[\frac{-20,906,000}{(20,909,280)^{2}}\right]^{2}(10)^{2} \\
& \sigma_{E F}=\sqrt{5.62848 * 10^{-17}+2.28658 * 10^{-13}}=0.000000478
\end{aligned}
$$

This means that a horizontal distance of 1000.000 feet reduced to the ellipsoid for these conditions computes to be 999.843 feet $+/-0.00048$ feet. Certainly the difference between horizontal and ellipsoid distance is significant, but the quality of the computed 
result does not appear to suffer significantly from using an approximate earth radius (+/1,000 feet) and an elevation known only to the nearest 10 feet.

\section{Metric Example:}

$$
\begin{aligned}
& \text { Assume: } \quad \mathrm{r}=6,372,000 \text { meters }+/-1,000 \text { meters } \\
& \mathrm{h}=1,000 \text { meters }+/-10 \text { meters } \\
& \text { Compute: } \quad E F=\frac{r}{r+h}=\frac{6,372,000}{6,373,000}=0.9998430881 \\
& \sigma_{E F}^{2}=\left[\frac{1,000}{(6,373,000)^{2}}\right]^{2}(1000)^{2}+\left[\frac{-6,372,000}{(6,373,000)^{2}}\right]^{2}(10)^{2} \\
& \sigma_{E F}=\sqrt{6.06211 * 10^{-16}+2.46136 * 10^{-12}}=0.000001569
\end{aligned}
$$

This means that a horizontal distance of 1000.000 meters reduced to sea level for these conditions computes to be 999.843 meters $+/-0.0016$ meters. Here too, the difference between horizontal and ellipsoid distance is significant, but the quality of the result does not appear to suffer significantly from using an approximate earth radius (+/- 1,000 meters) and an elevation known only to the nearest 10 meters.

Note in both examples that the contribution of uncertainty due to earth radius (first term under the square root symbol) is much smaller than the contribution due to uncertainty of elevation. Also note, the examples are different in that a 10 meter uncertainty in elevation in the metric example is much larger than the 10 feet uncertainty for elevation in the English unit example. 
Tabular Results - Metric:

Table 2 includes representative values of EF at five different elevations for a variety of elevation uncertainties and assuming uncertainty of earth radius from $1,000 \mathrm{~m}$ to 20,000 m. The relative uncertainty of the elevation factor for each combination is obtained by computing the reciprocal of the tabular entry. For example, the most precise entry is $0.0000001570(1: 6,369,000)$ in the upper left corner and the least precise entry is $0.0000157970(1: 63,300)$ in the lower right corner. Although Table 2 can be quite useful for general circumstances, a specific answer for any combination selected by the user is obtained using equation (6).

\section{Table 2, Standard Deviations of Elevation Factors for Various Combinations}

\begin{tabular}{cccccc}
$\begin{array}{c}\text { Elev. } \\
\text { meters }\end{array}$ & $\begin{array}{c}\text { Elev. } \\
\text { Sigma }\end{array}$ & $\begin{array}{c}\text { Radius Sigma } \\
1,000 \mathrm{~m}\end{array}$ & $\begin{array}{c}\text { Radius Sigma } \\
5,000 \mathrm{~m}\end{array}$ & $\begin{array}{c}\text { Radius Sigma } \\
10,000 \mathrm{~m}\end{array}$ & $\begin{array}{c}\text { Radius Sigma } \\
20,000 \mathrm{~m}\end{array}$ \\
\hline 100 & 1 & 0.0000001570 & 0.0000001574 & 0.0000001589 & 0.0000001645 \\
100 & 10 & 0.0000015693 & 0.0000015694 & 0.0000015695 & 0.0000015701 \\
100 & 50 & 0.0000078466 & 0.0000078466 & 0.0000078466 & 0.0000078467 \\
100 & 100 & 0.0000156932 & 0.0000156932 & 0.0000156932 & 0.0000156932 \\
& & & & & \\
500 & 1 & 0.0000001574 & 0.0000001686 & 0.0000001995 & 0.0000002920 \\
500 & 10 & 0.0000015692 & 0.0000015703 & 0.0000015739 & 0.0000015883 \\
500 & 50 & 0.0000078456 & 0.0000078458 & 0.0000078466 & 0.0000078495 \\
500 & 100 & 0.0000156912 & 0.0000156913 & 0.0000156917 & 0.0000156931 \\
& & & & & \\
1000 & 1 & 0.0000001588 & 0.0000001994 & 0.0000002920 & 0.0000005168 \\
1000 & 10 & 0.0000015691 & 0.0000015737 & 0.0000015881 & 0.0000016443 \\
1000 & 50 & 0.0000078444 & 0.0000078453 & 0.0000078482 & 0.0000078598 \\
1000 & 100 & 0.0000156888 & 0.0000156892 & 0.0000156907 & 0.0000156965 \\
& & & & & \\
2000 & 1 & 0.0000001644 & 0.0000002919 & 0.0000005167 & 0.0000009970 \\
2000 & 10 & 0.0000015692 & 0.0000015876 & 0.0000016438 & 0.0000018518 \\
2000 & 50 & 0.0000078421 & 0.0000078458 & 0.0000078573 & 0.0000079035 \\
2000 & 100 & 0.0000156839 & 0.0000156857 & 0.0000156915 & 0.0000157147 \\
& & & & & \\
4000 & 1 & 0.0000001851 & 0.0000005163 & 0.0000009963 & 0.0000019741 \\
4000 & 10 & 0.0000015705 & 0.0000016428 & 0.0000018506 & 0.0000025158 \\
4000 & 50 & 0.0000078376 & 0.0000078524 & 0.0000078985 & 0.0000080803 \\
4000 & 100 & 0.0000156743 & 0.0000156817 & 0.0000157048 & 0.0000157970
\end{tabular}




\section{Conclusions:}

Conclusions and final comments are:

1. The average earth radius commonly used is acceptable for all but the most demanding applications. Values of earth radius in Table 1 go from 6,356,752 m at the equator to $6,399,594 \mathrm{~m}$ at the pole. Using an average earth radius of $6,372,000 \mathrm{~m}$ and an earth radius uncertainty of 20,000 meters, the right-most column of Table 2 includes all latitudes from S $65^{\circ}$ to $\mathrm{N} 65^{\circ}$.

2. The accuracy of the elevation factor is not very sensitive to elevation itself. Table 2 includes elevation factors for elevations of $100,500,1000,2000$, and 4000 meters. The differences in each column for a given elevation sigma are quite small.

3. The accuracy of the computed elevation factor is affected by uncertainty in the elevation. But the level of sensitivity appears to be rather low. In fact, unless pursuing extremely accurate results, it appears one can use orthometric heights and ellipsoid heights interchangeably when making the elevation reduction. That means geoid modeling for purposes of elevation reduction is often moot. Table 2 includes and shows the impact of elevation uncertainties up to 100 meters. Few locations in the continental U.S. have geoid heights over 50 meters.

4. Regardless of the values shown in Table 2, the accuracy of the elevation factor for any set of conditions can be readily computed using equation 6 . It is probably more important to document the assumptions and values used on a given project or line reduction than it is to use one particular value or another.

5. Using the elevation factor is applying a systematic error correction. If a systematic error is not identified and corrected, the default consequence is treating systematic error as part of the random error budget. This paper treats 
the quality of the elevation factor as a random error. Nothing in this paper is meant to suggest that knowledgeable professionals should not evaluate systematic error sources and make the appropriate corrections.

\section{Symbols:}

$$
\begin{array}{ll}
\mathrm{EF} & =\text { Elevation Factor } \\
\mathrm{r} & =\text { Radius of earth } \\
\mathrm{h} & =\text { Elevation of horizontal distance } \\
\mathrm{a} & =\text { Semi-major axis of earth } \\
e^{2} & =\text { Eccentricity squared for the earth } \\
\phi & =\text { Geodetic latitude }
\end{array}
$$

\section{References:}

1. Burkholder, Earl F., 1991; "Computation of Horizontal/Level Distances", Journal of Surveying Engineering, Vol. 117, No. 3, pages 104 - 116.

2. Burkholder, Earl F., 2002, "Elevations and the Global Spatial Data Model (GSDM)," presented at the Institute of Navigation $58^{\text {th }}$ Annual Meeting, June 24-26, 2002, Albuquerque, NM. - www.zianet.com/globalcogo/elevgsdm.pdf 